\title{
Existence of Concave Positive Solutions for Boundary Value Problem of Nonlinear Fractional Differential Equation with $p$-Laplacian Operator
}

\author{
Jinhua Wang, Hongjun Xiang, and ZhiGang Liu \\ Department of Mathematics, Xiangnan University, Chenzhou 423000, China \\ Correspondence should be addressed to Hongjun Xiang, hunxhjxhj67@126.com
}

Received 27 July 2009; Accepted 9 March 2010

Academic Editor: Rodica Costin

Copyright (C) 2010 Jinhua Wang et al. This is an open access article distributed under the Creative Commons Attribution License, which permits unrestricted use, distribution, and reproduction in any medium, provided the original work is properly cited.

We consider the existence and multiplicity of concave positive solutions for boundary value problem of nonlinear fractional differential equation with $p$-Laplacian operator $D_{0+}^{\gamma}\left(\phi_{p}\left(D_{0+}^{\alpha} u(t)\right)\right)+$ $f\left(t, u(t), D_{0+}^{\rho} u(t)\right)=0,0<t<1, u(0)=u^{\prime}(1)=0, u^{\prime \prime}(0)=0,\left.D_{0+}^{\alpha} u(t)\right|_{t=0}=0$, where $0<\gamma<1$, $2<\alpha<3,0<\rho \leqslant 1, D_{0+}^{\alpha}$ denotes the Caputo derivative, and $f:[0,1] \times[0,+\infty) \times R \rightarrow[0,+\infty)$ is continuous function, $\phi_{p}(s)=|s|^{p-2} s, p>1,\left(\phi_{p}\right)^{-1}=\phi_{q}, 1 / p+1 / q=1$. By using fixed point theorem, the results for existence and multiplicity of concave positive solutions to the above boundary value problem are obtained. Finally, an example is given to show the effectiveness of our works.

\section{Introduction}

As we know, boundary value problems of integer-order differential equations have been intensively studied; see [1-5] and therein. Recently, due to the wide development of its theory of fractional calculus itself as well as its applications, fractional differential equations have been constantly attracting attention of many scholars; see, for example, [6-15].

In [7], Jafari and Gejji used the adomian decomposition method for solving the existence of solutions of boundary value problem:

$$
\begin{gathered}
D^{\alpha} u(t)+\mu f(t, u(t))=0, \quad 0<t<1,1<\alpha \leqslant 2, \\
u(0)=0, \quad u(1)=b .
\end{gathered}
$$


In [9], by using fixed point theorems on cones, Dehghani and Ghanbari considered triple positive solutions of nonlinear fractional boundary value problem:

$$
\begin{gathered}
D^{\alpha} x(t)+q(t) f\left(t, x(t), x^{\prime}(t)\right)=0, \quad 0<t<1,2 \leqslant \alpha<3, \\
x(0)=x(1)=0,
\end{gathered}
$$

where $D^{\alpha}$ is the standard Riemann-Liouvill derivative. But we think that Green's function in [9] is wrong; if $\alpha>2$, then, Green's function cannot be decided by $x(0)=x(1)=0$.

In [11], using fixed point theorems on cones, Zhang investigated the existence and multiplicity of positive solutions of the following problem:

$$
\begin{gathered}
D_{0+}^{\alpha} u(t)=f(t, u(t)), \quad 0<t<1,1<\alpha \leqslant 2, \\
u(0)+u^{\prime}(0)=0, \quad u(1)+u^{\prime}(1)=0
\end{gathered}
$$

where $D_{0+}^{\alpha}$ is the Caputo fractional derivative.

In [12], by means of Schauder fixed-point theorem, Su and Liu studied the existence of nonlinear fractional boundary value problem involving Caputo's derivative:

$$
\begin{gathered}
D_{0+}^{\alpha} u(t)=f\left(t, u(t), D_{0+}^{\beta} u(t)\right)=0, \quad 0<t<1,1<\alpha \leqslant 2, \\
u(0)=u^{\prime}(1)=0, \quad \text { or } \quad u^{\prime}(0)=u(1)=0, \quad \text { or } \quad u(0)=u(1)=0 .
\end{gathered}
$$

To the best of our knowledge, the existence of concave positive solutions of fractional order equation is seldom considered and investigated. Motivated by the above arguments, the main objective of this paper is to investigate the existence and multiplicity of concave positive solutions of boundary value problem of fractional differential equation with $p$ Laplacian operator as follows:

$$
\begin{gathered}
D_{0+}^{\gamma}\left(\phi_{p}\left(D_{0+}^{\alpha} u(t)\right)\right)+f\left(t, u(t), D_{0+}^{\rho} u(t)\right)=0, \quad 0<t<1, \\
u(0)=u^{\prime}(1)=0, \quad u^{\prime \prime}(0)=0,\left.\quad D_{0+}^{\alpha} u(t)\right|_{t=0}=0,
\end{gathered}
$$

where $0<\gamma<1,2<\alpha<3,0<\rho \leqslant 1, D_{0+}^{\alpha}$ denotes the Caputo derivative, and $f:[0,1] \times$ $[0,+\infty) \times R \rightarrow[0,+\infty)$ is continuous function, $\phi_{p}(s)=|s|^{p-2} s, p>1,\left(\phi_{p}\right)^{-1}=\phi_{q}, 1 / p+1 / q=1$.

By using fixed point theorem, some results for multiplicity of concave positive solutions to the above boundary value problems are obtained. Finally, an example is given to show the effectiveness of our works.

The rest of the paper is organized as follows. In Section 2, we will introduce some lemmas and definitions which will be used later. In Section 3, the multiplicity of concave positive solutions for the boundary value problem (1.5) will be discussed. 
International Journal of Mathematics and Mathematical Sciences

\section{Basic Definitions and Preliminaries}

Firstly we present here some necessary definitions and lemmas.

Definition 2.1 (see $[6,16,17]$ ). The fractional integral of order $\alpha>0$ of a function $y:(0, \infty) \rightarrow$ $R$ is given by

$$
I^{\alpha} y(t)=\frac{1}{\Gamma(\alpha)} \int_{0}^{t}(t-s)^{\alpha-1} y(s) d s,
$$

provided that the right side is pointwise defined on $(0, \infty)$.

Definition 2.2 (see $[6,16,17]$ ). The Riemann-Liouville fractional derivative of order $\alpha>0$ of a continuous function $y:(0, \infty) \rightarrow R$ is given by

$$
D^{\alpha} y(t)=\frac{1}{\Gamma(n-\alpha)}\left(\frac{d}{d t}\right)^{n} \int_{0}^{t}(t-s)^{n-\alpha-1} y(s) d s,
$$

where $n=[\alpha]+1$ provided that the right side is pointwise defined on $(0, \infty)$.

Definition 2.3 (see [17]). Caputo's derivative of order $\alpha>0$ of a function $y:(0, \infty) \rightarrow R$ is defined as

$$
D^{\alpha} y(t)=\frac{1}{\Gamma(n-\alpha)} \int_{0}^{t}(t-s)^{n-\alpha-1} y^{(n)}(s) d s, \quad n-1<\alpha<n,
$$

provided that the right side is pointwise defined on $(0, \infty)$.

Remark 2.4. The following properties are well known:

(1) $D_{0+}^{\alpha} D_{0+}^{\beta} y(t)=D_{0+}^{\alpha+\beta} y(t), \alpha>0, \beta>0$;

(2) $D_{0+}^{-\alpha} y(t)=I_{0+}^{\alpha} y(t), \alpha>0$;

(3) $I_{0+}^{\alpha}: C[0,1] \rightarrow C[0,1], \alpha>0$.

If $\alpha$ is an integer, the derivative for order $\alpha$ is understood in the sense of usual differentiation.

Definition 2.5. Let $E$ be a real Banach space over $R$. A nonempty convex closed set $P \subset E$ is said to be a cone provided that

(a) $a u \in P$, for all $u \in P, a \geqslant 0$;

(b) $u,-u \in P$, implies $u=0$. 
Definition 2.6. Let $E$ be a real Banach space and let $P \subset E$ be a cone. A function $\psi: P \rightarrow[0, \infty)$ is called a nonegative continuous concave functional if $\psi$ is continuous and

$$
\psi(\lambda x+(1-\lambda) y) \geqslant \lambda \psi(x)+(1-\lambda) \psi(y)
$$

for all $x, y \in P$ and $0 \leqslant \lambda \leqslant 1$.

Definition 2.7. Let $E$ be a real Banach space and let $P \subset E$ be a cone. A function $\varphi: P \rightarrow[0, \infty)$ is called a nonegative continuous convex functional if $\varphi$ is continuous and

$$
\varphi(\lambda x+(1-\lambda) y) \leqslant \lambda \varphi(x)+(1-\lambda) \varphi(y)
$$

for all $x, y \in P$ and $0 \leqslant \lambda \leqslant 1$.

Suppose that $\varphi, \theta: P \rightarrow[0,+\infty)$ are two nonnegative continuous convex functionals satisfying

$$
\|u\| \leqslant M \max \{\varphi(u), \theta(u)\}, \quad \text { for } u \in P,
$$

where $M$ is a positive constant and

$$
\Omega=\{u \in P \mid \varphi(u)<r, \theta(u)<L\} \neq \varnothing, \quad \text { for } r>0, L>0 .
$$

Let $r>a>0, L>0$ be given constants, let $\varphi, \theta: P \rightarrow[0,+\infty)$ be two nonnegative continuous convex functionals satisfying (2.6) and (2.7), and let $\psi$ be a nonnegative continuous concave functional on the cone $P$. Define the bounded convex sets:

$$
\begin{gathered}
P(\varphi, r ; \theta, L)=\{u \in P \mid \varphi(u)<r, \theta(u)<L\}, \\
\bar{P}(\varphi, r ; \theta, L)=\{u \in P \mid \varphi(u) \leqslant r, \theta(u) \leqslant L\}, \\
P(\varphi, r ; \theta, L ; \psi, a)=\{u \in P \mid \varphi(u)<r, \theta(u)<L, \psi(u)>a\}, \\
\bar{P}(\varphi, r ; \theta, L ; \psi, a)=\{u \in P \mid \varphi(u) \leqslant r, \theta(u) \leqslant L, \psi(u) \geqslant a\} .
\end{gathered}
$$

Lemma 2.8 (see $[2,5]$ ). Let $E$ be a Banach space, let $P \subset E$ be a cone, and let $r_{2} \geqslant c>b>r_{1}>0$, $L_{2} \geqslant L_{1}>0$ be given constants. Assume that $\varphi, \theta$ are two nonnegative continuous convex functionals on $P$, such that (2.6) and (2.7) are satisfied; let $\psi$ be a nonnegative continuous concave functional on $P$, such that $\psi(u) \leqslant \varphi(u)$ for all $u \in \bar{P}\left(\varphi, r_{2} ; \theta, L_{2}\right)$ and let $T: \bar{P}\left(\varphi, r_{2} ; \theta, L_{2}\right) \rightarrow \bar{P}\left(\varphi, r_{2} ; \theta, L_{2}\right)$ be 
a completely continuous operator. Suppose the following:

$\left(C_{1}\right) \quad\left\{u \in \bar{P}\left(\varphi, c ; \theta, L_{2} ; \psi, b\right) \mid \psi(u)>b\right\} \neq \varnothing, \psi(T u)>$ b for all $u \in \bar{P}\left(\varphi, c ; \theta, L_{2} ; \psi, b\right)$;

$\left(C_{2}\right) \varphi(T u)<r_{1}, \theta(T u)<L_{1}$, for all $u \in \bar{P}\left(\varphi, r_{1} ; \theta, L_{1}\right)$;

$\left(C_{3}\right) \psi(T u)>b$, for all $u \in \bar{P}\left(\varphi, r_{2} ; \theta, L_{2} ; \psi, b\right)$ with $\varphi(T u)>c$.

Then $T$ has at least three fixed points $u_{1}, u_{2}$, and $u_{3}$ in $\bar{P}\left(\varphi, r_{2} ; \theta, L_{2}\right)$. Further $u_{1} \in P(\varphi$, $\left.r_{1} ; \theta, L_{1}\right), u_{2} \in\left\{\bar{P}\left(\varphi, r_{2} ; \theta, L_{2} ; \psi, b\right) \mid \psi(u)>b\right\}$, and $u_{3} \in \bar{P}\left(\varphi, r_{2} ; \theta, L_{2}\right) \backslash\left(\bar{P}\left(\varphi, r_{2} ; \theta, L_{2} ;\right.\right.$ $\psi, b) \cup \bar{P}\left(\varphi, r_{1} ; \theta, L_{1}\right)$.

Lemma 2.9 (see [12]). Assume that $u \in C(0,1) \cap L(0,1)$ with a fractional derivative of order $\alpha$ that belongs to $C(0,1) \cap L(0,1)$. Then

$$
I_{0+}^{\alpha} D_{0+}^{\alpha} u(t)=u(t)+C_{0}+C_{1} t+\cdots+C_{n-1} t^{n-1},
$$

for some $C_{i} \in R, i=0,1, \ldots, n-1$, where $n$ is the smallest integer greater than or equal to $\alpha$.

Lemma 2.10. Let $y \in C[0,1]$; then the boundary value problem

$$
\begin{gathered}
D_{0+}^{\alpha} u(t)+y(t)=0, \quad 0<t<1,2<\alpha<3, \\
u(0)=u^{\prime}(1)=u^{\prime \prime}(0)=0
\end{gathered}
$$

has a unique solution

$$
u(t)=\int_{0}^{1} G(t, s) y(s) d s,
$$

where

$$
G(t, s)= \begin{cases}\frac{(\alpha-1) t(1-s)^{\alpha-2}-(t-s)^{\alpha-1}}{\Gamma(\alpha)}, & 0 \leqslant s \leqslant t \leqslant 1, \\ \frac{(\alpha-1) t(1-s)^{\alpha-2}}{\Gamma(\alpha)}, & 0 \leqslant t \leqslant s \leqslant 1 .\end{cases}
$$

Proof. We may apply Lemma 2.9 to reduce (2.10) to an equivalent integral equation:

$$
u(t)=-\frac{1}{\Gamma(\alpha)} \int_{0}^{t}(t-s)^{\alpha-1} y(s) d s+C_{0}+C_{1} t+C_{2} t^{2}
$$


From (2.14), we have

$$
\begin{aligned}
& u^{\prime}(t)=-\frac{1}{\Gamma(\alpha)} \int_{0}^{t}(\alpha-1)(t-s)^{\alpha-2} y(s) d s+C_{1}+2 C_{2} t \\
& u^{\prime \prime}(t)=-\frac{1}{\Gamma(\alpha)} \int_{0}^{t}(\alpha-1)(\alpha-2)(t-s)^{\alpha-3} y(s) d s+2 C_{2}
\end{aligned}
$$

and by (2.10) and (2.11), there are $C_{0}=C_{2}=0, C_{1}=(1 / \Gamma(\alpha)) \int_{0}^{1}(\alpha-1)(1-s)^{\alpha-2} y(s) d s$.

Therefore, the unique solution of problem (2.10) and (2.11) is

$$
\begin{aligned}
u(t) & =-\frac{1}{\Gamma(\alpha)} \int_{0}^{t}(t-s)^{\alpha-1} y(s) d s+\frac{1}{\Gamma(\alpha)} \int_{0}^{1}(\alpha-1) t(1-s)^{\alpha-2} y(s) d s \\
& =\int_{0}^{t} \frac{(\alpha-1) t(1-s)^{\alpha-2}-(t-s)^{\alpha-1}}{\Gamma(\alpha)} y(s) d s+\int_{t}^{1} \frac{(\alpha-1) t(1-s)^{\alpha-2}}{\Gamma(\alpha)} y(s) d s \\
& =\int_{0}^{1} G(t, s) y(s) d s .
\end{aligned}
$$

Lemma 2.11. The function $G(t, s)$ defined by (2.13) satisfies the following conditions:

(1) $G(t, s) \geqslant 0, G(t, s) \leqslant G(1, s)$ for $0 \leqslant t, s \leqslant 1$,

(2) $G(t, s) \geqslant t^{\alpha-1} G(1, s)$, for $0 \leqslant t, s \leqslant 1$.

Proof. Since

$$
\begin{aligned}
g(t, s) & =:(\alpha-1) t(1-s)^{\alpha-2}-(t-s)^{\alpha-1} \\
& \geqslant(\alpha-1) t^{\alpha-1}(1-s)^{\alpha-2}-(t-s)^{\alpha-1} \\
& =t^{\alpha-1}\left[(\alpha-1)(1-s)^{\alpha-2}-\left(1-\frac{s}{t}\right)^{\alpha-1}\right] \\
& \geqslant t^{\alpha-1}\left[(\alpha-1)(1-s)^{\alpha-2}-(1-s)^{\alpha-1}\right] \\
& \geqslant 0, \quad \text { for } 2<\alpha<3,0 \leqslant s \leqslant t \leqslant 1
\end{aligned}
$$

observing (2.13), we have $G(t, s) \geqslant 0$. 
Form (2.13), we obtain

$$
G_{t}^{\prime}(t, s)= \begin{cases}\frac{(\alpha-1)(1-s)^{\alpha-2}-(\alpha-1)(t-s)^{\alpha-2}}{\Gamma(\alpha)}, & 0 \leqslant s \leqslant t \leqslant 1 \\ \frac{(\alpha-1)(1-s)^{\alpha-2}}{\Gamma(\alpha)}, & 0 \leqslant t \leqslant s \leqslant 1 .\end{cases}
$$

Clearly, $G_{t}^{\prime}(t, s) \geqslant 0$, for $0 \leqslant t, s \leqslant 1$, we have that $G(t, s)$ is increasing with respect to $t \in[0,1]$, and therefore, $G(t, s) \leqslant G(1, s)$, for $0 \leqslant t, s \leqslant 1$. (1) of Lemma 2.11 holds.

On the other hand, if $t \geqslant s$, then,

$$
\begin{aligned}
\frac{G(t, s)}{G(1, s)} & =\frac{(\alpha-1) t(1-s)^{\alpha-2}-(t-s)^{\alpha-1}}{(\alpha-1)(1-s)^{\alpha-2}-(1-s)^{\alpha-1}} \\
& \geqslant \frac{t^{\alpha-1}\left[(\alpha-1)(1-s)^{\alpha-2}-(1-s)^{\alpha-1}\right]}{(\alpha-1)(1-s)^{\alpha-2}-(1-s)^{\alpha-1}} \\
& =t^{\alpha-1} .
\end{aligned}
$$

If $t \leqslant s$, then $G(t, s) / G(1, s)=t^{\alpha-1}$; therefore, $G(t, s) \geqslant t^{\alpha-1} G(1, s)$, for $0 \leqslant s, t \leqslant 1$. (2) of Lemma 2.11 holds.

\section{Existence of Three Concave Positive Solutions}

In this section, we study the existence of concave positive solution for problem (1.5).

Let $E=C^{1}[0,1]$. From Definitions 2.1 and 2.3, we obtain $D_{0+}^{\rho} u(t)=I_{0+}^{1-\rho} u^{\prime}(t), 0<\rho<1$, and $D_{0+}^{\rho} u(t)=u^{\prime}(t), \rho=1$. So, by (3) of Remark 2.4, we know that $D_{0+}^{\rho} u(t)$ is continuous for all $u(t) \in E$. Hence, for all $u(t) \in E$, we can define

$$
\|u\|= \begin{cases}\max _{0 \leqslant t \leqslant 1}|u(t)|+\max _{0 \leqslant t \leqslant 1}\left|D_{0+}^{\rho} u(t)\right|+\max _{0 \leqslant t \leqslant 1}\left|u^{\prime}(t)\right|, & 0<\rho<1, \\ \max _{0 \leqslant t \leqslant 1}|u(t)|+\max _{0 \leqslant t \leqslant 1}\left|u^{\prime}(t)\right|, & \rho=1 .\end{cases}
$$

Lemma 3.1 (see $[12]) .(E,\|\cdot\|)$ is a Banach space.

Define the cone $P \subset E$ by $P=\{u \in E \mid u(t) \geqslant 0, u(t)$ is concave on $[0,1]\}$. 
Let the nonnegative continuous concave functional $\psi$ and the nonnegative continuous convex functionals $\varphi, \theta$ be defined on the cone $P$ by

$$
\begin{gathered}
\psi(u)=\min _{1 / k \leqslant t \leqslant(k-1) / k}|u(t)|, \quad \varphi(u)=\max _{0 \leqslant t \leqslant 1}|u(t)|, \\
\theta(u)= \begin{cases}\max _{0 \leqslant t \leqslant 1}\left|D_{0+}^{\rho} u(t)\right|+\max _{0 \leqslant t \leqslant 1}\left|u^{\prime}(t)\right|, & 0<\rho<1, \\
\max _{0 \leqslant t \leqslant 1}\left|u^{\prime}(t)\right|, & \rho=1 .\end{cases}
\end{gathered}
$$

Lemma 3.2 (see [1]). Let $u \in P, k \geqslant 3$; then

$$
\min _{1 / k \leqslant t \leqslant(k-1) / k}|u(t)| \geqslant \frac{1}{k} \max _{0 \leqslant t \leqslant 1}|u(t)|
$$

Lemma 3.3. BVP (1.5) is equivalent to the integral equation

$$
u(t)=\int_{0}^{1} G(t, s) \phi_{q}\left(\frac{1}{\Gamma(\gamma)} \int_{0}^{s}(s-\tau)^{\gamma-1} f\left(\tau, u(\tau), D_{0+}^{\rho} u(\tau)\right) d \tau\right) d s
$$

Proof. From BVP (1.5) and Lemma 2.9, we have

$$
\begin{aligned}
\phi_{p}\left(D_{0+}^{\alpha} u(t)\right) & =-I_{0+}^{\gamma} f\left(t, u(t), D_{0+}^{\rho} u(t)\right)+C, \\
& =-\frac{1}{\Gamma(\gamma)} \int_{0}^{t}(t-\tau)^{\gamma-1} f\left(\tau, u(\tau), D_{0+}^{\rho} u(\tau)\right) d \tau+C .
\end{aligned}
$$

By $\left.D_{0+}^{\alpha} u(t)\right|_{t=0}=0$, there is $C=0$, and then,

$$
D_{0+}^{\alpha} u(t)=-\phi_{q}\left(\frac{1}{\Gamma(\gamma)} \int_{0}^{t}(t-\tau)^{\gamma-1} f\left(\tau, u(\tau), D_{0+}^{\rho} u(\tau)\right) d \tau\right) .
$$

Therefore, BVP(1.5) is equivalent to following problem:

$$
\begin{gathered}
D_{0+}^{\alpha} u(t)+\phi_{q}\left(\frac{1}{\Gamma(\gamma)} \int_{0}^{t}(t-\tau)^{\gamma-1} f\left(\tau, u(\tau), D_{0+}^{\rho} u(\tau)\right) d \tau\right)=0, \quad 0<t<1,2<\alpha<3, \\
u(0)=u^{\prime}(1)=u^{\prime \prime}(0)=0 .
\end{gathered}
$$

By Lemma 2.10, BVP (1.5) is equivalent to the integral equation (3.4). 
International Journal of Mathematics and Mathematical Sciences

Let $T: P \rightarrow E$ be the operator defined by

$$
T u(t)=\int_{0}^{1} G(t, s) \phi_{q}\left(\frac{1}{\Gamma(\gamma)} \int_{0}^{s}(s-\tau)^{\gamma-1} f\left(\tau, u(\tau), D_{0+}^{\rho} u(\tau)\right) d \tau\right) d s=: F(t)
$$

Lemma 3.4. $T: P \rightarrow P$ is completely continuous.

Proof. Let $u \in P$; in view of nonnegativeness and continuity of $G(t, s)$ and $f(t, u, v)$, we have $T u \geqslant 0$, and $t \in[0,1]$ is continuous:

$$
\begin{aligned}
(T u)^{\prime}(t)= & \int_{0}^{1} G_{t}^{\prime}(t, s) \phi_{q}\left(\frac{1}{\Gamma(\gamma)} \int_{0}^{s}(s-\tau)^{\gamma-1} f\left(\tau, u(\tau), D_{0+}^{\rho} u(\tau)\right) d \tau\right) d s \\
= & \int_{0}^{t} \frac{(\alpha-1)(1-s)^{\alpha-2}-(\alpha-1)(t-s)^{\alpha-2}}{\Gamma(\alpha)} \phi_{q}\left(\frac{1}{\Gamma(\gamma)} \int_{0}^{s}(s-\tau)^{\gamma-1} f\left(\tau, u(\tau), D_{0+}^{\rho} u(\tau)\right) d \tau\right) d s \\
& +\int_{t}^{1} \frac{(\alpha-1)(1-s)^{\alpha-2}}{\Gamma(\alpha)} \phi_{q}\left(\frac{1}{\Gamma(\gamma)} \int_{0}^{s}(s-\tau)^{\gamma-1} f\left(\tau, u(\tau), D_{0+}^{\rho} u(\tau)\right) d \tau\right) d s .
\end{aligned}
$$

Clearly, $(T u)^{\prime}(t)$ is continuous for $\alpha<2$.

By Remark 2.4 and noting (3.4) and (3.6), we have

$$
\begin{aligned}
D_{0+}^{2}(T u)(t) & =D_{0+}^{2-\alpha} D_{0+}^{\alpha}(T u)(t) \\
& =D_{0+}^{2-\alpha}\left(D_{0+}^{\alpha} F(t)\right) \\
& =-D_{0+}^{2-\alpha}\left(\phi_{q}\left(\frac{1}{\Gamma(\gamma)} \int_{0}^{t}(t-\tau)^{\gamma-1} f\left(\tau, u(\tau), D_{0+}^{\rho} u(\tau)\right) d \tau\right)\right) \\
& =-I_{0+}^{\alpha-2}\left(\phi_{q}\left(\frac{1}{\Gamma(\gamma)} \int_{0}^{t}(t-\tau)^{\gamma-1} f\left(\tau, u(\tau), D_{0+}^{\rho} u(\tau)\right) d \tau\right)\right) \\
& =-\frac{1}{\Gamma(\alpha-2)} \int_{0}^{t}(t-s)^{\alpha-3}\left(\phi_{q}\left(\frac{1}{\Gamma(\gamma)} \int_{0}^{t}(t-\tau)^{\gamma-1} f\left(\tau, u(\tau), D_{0+}^{\rho} u(\tau)\right) d \tau\right) d s\right) \\
& \leqslant 0 .
\end{aligned}
$$

So, $T u$ is concave on $[0,1]$ and $T u \in C^{1}[0,1]$; we obtain $T(P) \subset P$. 
10

International Journal of Mathematics and Mathematical Sciences

Let $\Omega \in P$ be bounded; that is, there exists a positive constant $M>0$ such that $\|u\| \leqslant M$ for all $u \in \Omega$.

Let $N=\max _{(t, u, v) \in[0,1] \times[0, M] \times[-M, M]} \mid f\left(t, u(t), D_{0+}^{\rho} u(t) \mid\right.$; then, for all $u \in \Omega$, we have

$$
\begin{aligned}
& |T u(t)|=\left|\int_{0}^{1} G(t, s) \phi_{q}\left(\frac{1}{\Gamma(\gamma)} \int_{0}^{s}(s-\tau)^{\gamma-1} f\left(\tau, u(\tau), D_{0+}^{\rho} u(\tau)\right) d \tau\right) d s\right| \\
& \leqslant\left(\frac{N}{\Gamma(\gamma+1)}\right)^{q-1} \int_{0}^{1} G(1, s) d s \\
& =\left(\frac{N}{\Gamma(\gamma+1)}\right)^{q-1}\left[\frac{1}{\Gamma(\alpha)}-\frac{1}{\Gamma(\alpha+1)}\right] \\
& \leqslant\left(\frac{N}{\Gamma(\gamma+1)}\right)^{q-1} \frac{1}{\Gamma(\alpha)} \\
& \left|T u^{\prime}(t)\right|=\left|\int_{0}^{1} G_{t}^{\prime}(t, s) \phi_{q}\left(\frac{1}{\Gamma(\gamma)} \int_{0}^{s}(s-\tau)^{\gamma-1} f\left(\tau, u(\tau), D_{0+}^{\rho} u(\tau)\right) d \tau\right) d s\right| \\
& \leqslant\left(\frac{N}{\Gamma(\gamma+1)}\right)^{q-1} \int_{0}^{1} G_{t}^{\prime}(t, s) d s \\
& \leqslant\left(\frac{N}{\Gamma(\gamma+1)}\right)^{q-1} \frac{1}{\Gamma(\alpha)} \\
& \left|D_{0+}^{\rho}(T u)(t)\right|=\left|\frac{1}{\Gamma(1-\rho)} \int_{0}^{t}(t-s)^{-\rho}(T u)^{\prime}(s) d s\right| \\
& \leqslant \frac{1}{\Gamma(1-\rho)} \int_{0}^{t}(t-s)^{-\rho} \\
& \times\left|\int_{0}^{1} G_{s}^{\prime}(s, v) \phi_{q}\left(\frac{1}{\Gamma(\gamma)} \int_{0}^{v}(v-\tau)^{\gamma-1} f\left(\tau, u(\tau), D_{0+}^{\rho} u(\tau)\right) d \tau\right)\right| d s \\
& \leqslant \frac{1}{\Gamma(1-\rho)}\left(\frac{N}{\Gamma(\gamma+1)}\right)^{q-1} \frac{1}{\Gamma(\alpha)} \int_{0}^{t}(t-s)^{-\rho} d s \\
& =\left(\frac{N}{\Gamma(\gamma+1)}\right)^{q-1} \frac{t^{1-\rho}}{(1-\rho) \Gamma(1-\rho) \Gamma(\alpha)} \\
& \leqslant\left(\frac{N}{\Gamma(\gamma+1)}\right)^{q-1} \frac{1}{\Gamma(2-\rho) \Gamma(\alpha)}
\end{aligned}
$$


So, for all $u \in \Omega,\|T u(t)\| \leqslant(N / \Gamma(\gamma+1))^{q-1}[2 / \Gamma(\alpha)+1 / \Gamma(2-\rho) \Gamma(\alpha)]$. Hence, $T(\Omega)$ is uniformly bounded.

Since $G(t, s)$ is continuous on $[0,1] \times[0,1]$, it is uniformly continuous on $[0,1] \times[0,1]$. Thus for fixed $s \in[0,1]$ and for any $\varepsilon>0$, there exists a constant $\delta>0$, such that any $t_{1}, t_{2} \in[0,1]$ and $\left|t_{1}-t_{2}\right|<\delta$,

$$
\left|G\left(t_{1}, s\right)-G\left(t_{2}, s\right)\right|<\varepsilon\left(\frac{\Gamma(\gamma+1)}{N}\right)^{q-1} .
$$

Therefore,

$$
\begin{aligned}
\left|T u\left(t_{2}\right)-T u\left(t_{1}\right)\right| & \leqslant \int_{0}^{1}\left|G\left(t_{2}, s\right)-G\left(t_{1}, s\right)\right| \phi_{q}\left(\frac{1}{\Gamma(\gamma)} \int_{0}^{s}(s-\tau)^{\gamma-1} f\left(\tau, u(\tau), D_{0+}^{\rho} u(\tau)\right) d \tau\right) d s \\
& \leqslant\left(\frac{N}{\Gamma(\gamma+1)}\right)^{q-1} \int_{0}^{1}\left|G\left(t_{2}, s\right)-G\left(t_{1}, s\right)\right| d s<\varepsilon .
\end{aligned}
$$

That is to say, $T(\Omega)$ is equicontinuous. By the means of the Arzela-Ascoli Theorem, we have that $T: P \rightarrow P$ is completely continuous. The proof is completed.

Let

$$
\begin{gathered}
A=\left(\frac{1}{\Gamma(\gamma+1)}\right)^{q-1} \frac{1}{\Gamma(\alpha)}, \\
B=\left(\frac{1}{k}\right)^{\gamma(q-1)}\left(\frac{1}{\Gamma(\gamma+1)}\right)^{q-1} \int_{1 / k}^{1} G(1, s) d s, \\
M=\left(\frac{1}{\Gamma(\gamma+1)}\right)^{q-1} \frac{1}{\Gamma(\alpha) \Gamma(2-\rho)} .
\end{gathered}
$$

Theorem 3.5. Suppose that there exist constants $0<r_{1}<b<k b \leqslant r_{2}, L_{2} \geqslant L_{1}>0$, such that $k b / B \leqslant \min \left(r_{2} / A, L_{2} / 2 A, L_{2} / 2 M\right)$, and the following conditions hold:

$\left(H_{1}\right) f(t, u, v)<\min \left\{\phi_{p}\left(r_{1} / A\right), \phi_{p}\left(L_{1} / 2 A\right), \phi_{p}\left(L_{1} / 2 M\right)\right\}$, for $(t, u, v) \in[0,1] \times\left[0, r_{1}\right] \times$ $\left[-L_{1}, L_{1}\right]$

$\left(H_{2}\right) f(t, u, v)>\phi_{p}(k b / B)$, for $(t, u, v) \in[1 / k, 1] \times[b, k b] \times\left[-L_{2}, L_{2}\right]$;

$\left(H_{3}\right) f(t, u, v) \leqslant \min \left\{\phi_{p}\left(r_{2} / A\right), \phi_{p}\left(L_{2} / 2 A\right), \phi_{p}\left(L_{2} / 2 M\right)\right\}$, for $(t, u, v) \in[0,1] \times\left[0, r_{2}\right] \times$ $\left[-L_{2}, L_{2}\right]$. 
Then, the BVP (1.5) has at least three concave positive solutions $u_{1}, u_{2}$, and $u_{3}$, such that

$$
\begin{gathered}
\max _{0 \leqslant t \leqslant 1}\left|u_{1}(t)\right|<r_{1}, \quad \max _{0 \leqslant t \leqslant 1}\left|D_{0+}^{\rho} u_{1}(t)\right|+\max _{0 \leqslant t \leqslant 1}\left|u_{1}^{\prime}(t)\right|<L_{1}, \\
b<\min _{1 / k \leqslant t \leqslant(k-1) / k}\left|u_{2}(t)\right|<\max _{0 \leqslant t \leqslant 1}\left|u_{2}(t)\right| \leqslant r_{2} \\
\max _{0 \leqslant t \leqslant 1}\left|D_{0+}^{\rho} u_{2}(t)\right|+\max _{0 \leqslant t \leqslant 1}\left|u_{2}^{\prime}(t)\right|<L_{2}, \\
r_{1}<\max _{0 \leqslant t \leqslant 1}\left|u_{3}(t)\right| \leqslant k b, \quad \min _{1 / k \leqslant t \leqslant(k-1) / k}\left|u_{3}(t)\right|<b, \\
L_{1}<\max _{0 \leqslant t \leqslant 1}\left|D_{0+}^{\rho} u_{3}(t)\right|+\max _{0 \leqslant t \leqslant 1}\left|u_{3}^{\prime}(t)\right|<L_{2} .
\end{gathered}
$$

Proof. By Lemmas 3.3 and 3.4, we have that $T: P \rightarrow P$ is completely continuous and problem (1.5) has a solution $u=u(t)$ if and only if $u(t)$ satisfies the operator equation $u=T u$.

Now, we show that all the conditions of Lemma 2.8 hold.

Step 1. We will show that $T: \bar{P}\left(\varphi, r_{2} ; \theta, L_{2}\right) \rightarrow \bar{P}\left(\varphi, r_{2} ; \theta, L_{2}\right)$.

If $u \in \bar{P}\left(\varphi, r_{2} ; \theta, L_{2}\right)$, then $\varphi(u) \leqslant r_{2}, \theta(u) \leqslant L_{2}$.

From $\left(H_{3}\right)$, we have

$$
\begin{aligned}
\varphi(T u(t)) & =\max _{0 \leq t \leq 1}|(T u)(t)| \\
& =\max _{0 \leq t \leq 1}\left|\int_{0}^{1} G(t, s) \phi_{q}\left(\frac{1}{\Gamma(r)} \int_{0}^{s}(s-\tau)^{r-1} f\left(\tau, u(\tau), D_{0+}^{\rho} u(\tau)\right) d \tau\right) d s\right| \\
& \leqslant \frac{r_{2}}{A} \int_{0}^{1} G(1, s) \phi_{q}\left(\frac{1}{\Gamma(r)} \int_{0}^{s}(s-\tau)^{r-1} d \tau\right) d s \\
& \leqslant \frac{r_{2}}{A}\left[\frac{1}{r \Gamma(r)}\right]^{q-1}\left(\frac{1}{\Gamma(\alpha)}-\frac{1}{\Gamma(\alpha+1)}\right) \\
& \leqslant \frac{r_{2}}{A}\left(\frac{1}{\Gamma(r+1)}\right)^{q-1} \frac{1}{\Gamma(\alpha)} \\
& =r_{2}
\end{aligned}
$$




$$
\begin{aligned}
& \max _{0 \leq t \leq 1}\left|(T u)^{\prime}(t)\right|=\max _{0 \leq t \leq 1}\left|\int_{0}^{1} G_{t}^{\prime}(t, s) \phi_{q}\left(\frac{1}{\Gamma(r)} \int_{0}^{s}(s-\tau)^{r-1} f\left(\tau, u(\tau), D_{0+}^{\rho} u(\tau)\right) d \tau\right) d s\right| \\
& \leqslant \frac{L_{2}}{2 A}\left[\frac{1}{\Gamma(\gamma+1)}\right]^{q-1} \int_{0}^{1} G_{t}^{\prime}(t, s) d s \\
& =\frac{L_{2}}{2 A}\left(\frac{1}{\Gamma(r+1)}\right)^{q-1} \frac{1}{\Gamma(\alpha)} \\
& \leqslant \frac{L_{2}}{2} \\
& \max _{0 \leq t \leq 1}\left|D_{0+}^{\rho}(T u)(t)\right|=\max _{0 \leq t \leq 1}\left|\frac{1}{\Gamma(1-\rho)} \int_{0}^{t}(t-s)^{-\rho}(T u)^{\prime}(s) d s\right| \\
& \leqslant \frac{1}{\Gamma(1-\rho)} \int_{0}^{t}(t-s)^{-\rho} \\
& \times\left|\int_{0}^{1} G_{s}^{\prime}(s, v) \phi_{q}\left(\frac{1}{\Gamma(\gamma)} \int_{0}^{v}(v-\tau)^{\gamma-1} f\left(\tau, u(\tau), D_{0+}^{\rho} u(\tau)\right) d \tau\right)\right| d s \\
& \leqslant \frac{L_{2}}{2 M} \frac{1}{\Gamma(1-\rho)}\left(\frac{1}{\Gamma(\gamma+1)}\right)^{q-1} \frac{1}{\Gamma(\alpha)} \int_{0}^{t}(t-s)^{-\rho} d s \\
& =\frac{L_{2}}{2 M}\left(\left(\frac{1}{\Gamma(\gamma+1)}\right)^{q-1} \frac{1}{\Gamma(\alpha) \Gamma(2-\rho)}\right) \\
& \leqslant \frac{L_{2}}{2} \text {. }
\end{aligned}
$$

Then, $\theta((T u)(t)) \leqslant L_{2}$.

So, $T: \bar{P}\left(\varphi, r_{2} ; \theta, L_{2}\right) \rightarrow \bar{P}\left(\varphi, r_{2} ; \theta, L_{2}\right)$.

Step 2. Let $u(t)=k b / 2,0 \leqslant t \leqslant 1$. It is easy to see that $u(t)=k b / 2 \in \bar{P}\left(\varphi, k b ; \theta, L_{2}, \psi, b\right)$, and $\psi(u)=\psi(k b / 2)>b$. Consequently, $\left\{u \in \bar{P}\left(\varphi, k b ; \theta, L_{2}, \psi, b\right) \mid \psi(u)>b\right\} \neq \varnothing$.

If $u \in \bar{P}\left(\varphi, k b ; \theta, L_{2}, \psi, b\right)$, then for all $1 / k \leqslant t \leqslant 1, b \leqslant u(t) \leqslant k b, \theta(u(t)) \leqslant L_{2}$. By $\left(H_{2}\right)$, we obtain $f\left(t, u(t), D_{0+}^{\rho} u(t)\right)>\phi_{p}(k b / B)$, for $1 / k \leqslant t \leqslant 1$. 
From Lemma 3.2, we have

$$
\begin{aligned}
\psi(T u) & =\min _{1 / k \leqslant t \leqslant(k-1) / k}|T u(t)| \\
& \geqslant \frac{1}{k} \max _{0 \leqslant t \leqslant 1}|(T u)(t)| \\
& =\frac{1}{k} \max _{0 \leqslant t \leqslant 1}\left|\int_{0}^{1} G(t, s) \phi_{q}\left(\frac{1}{\Gamma(r)} \int_{0}^{s}(s-\tau)^{r-1} f\left(\tau, u(\tau), D_{0+}^{\rho} u(\tau)\right) d \tau\right) d s\right| \\
& >\frac{k b}{B} \frac{1}{k} \max _{0 \leqslant t \leqslant 1} t^{\alpha-1} \int_{0}^{1} G(1, s) \phi_{q}\left(\frac{s^{\gamma}}{\gamma \Gamma(\gamma)}\right) d s \\
& =\frac{b}{B}\left(\frac{1}{\Gamma(\gamma+1)}\right)^{q-1} \int_{0}^{1} G(1, s) \phi_{q}\left(s^{\gamma}\right) d s \\
& >\frac{b}{B}\left(\frac{1}{k}\right)^{\gamma(q-1)}\left(\frac{1}{\Gamma(\gamma+1)}\right)^{q-1} \int_{1 / k}^{1} G(1, s) d s=b ;
\end{aligned}
$$

that is, $\psi(T u)>b$, for all $u \in \bar{P}\left(\varphi, k b ; \theta, L_{2}, \psi, b\right)$. This shows that condition $\left(C_{1}\right)$ of Lemma 2.8 holds.

Step 3. Let $u \in \bar{P}\left(\varphi, r_{1} ; \theta, L_{1}\right)$; by $\left(H_{1}\right)$, we have

$$
f\left(t, u(t), D_{0+}^{\rho} u(t)\right)<\min \left\{\phi_{p}\left(\frac{r_{1}}{A}\right), \phi_{p}\left(\frac{L_{1}}{2 A}\right), \phi_{p}\left(\frac{L_{1}}{2 M}\right)\right\} .
$$

Similarly, we can prove that $T: \bar{P}\left(\varphi, r_{1} ; \theta, L_{1}\right) \rightarrow \bar{P}\left(\varphi, r_{1} ; \theta, L_{1}\right) .\left(C_{2}\right)$ of Lemma 2.8 holds.

Step 4. Let $u \in \bar{P}\left(\varphi, k b ; \theta, L_{2} ; \psi, b\right)$, and $\varphi(T u)>k b$; we have

$$
\psi(T u)=\min _{1 / k \leqslant t \leqslant(k-1) / k}|T u(t)| \geqslant \frac{1}{k} \max _{0 \leqslant t \leqslant 1}|T u(t)|=\frac{1}{k} \varphi(T u)>b .
$$

$\left(C_{3}\right)$ of Lemma 2.8 holds. Therefore, the BVP (1.5) has at least three positive solutions $u_{1}, u_{2}$, and $u_{3}$ satisfying

$$
\begin{gathered}
\max _{0 \leqslant t \leqslant 1}\left|u_{1}(t)\right| \leqslant r_{1}, \quad \max _{0 \leqslant t \leqslant 1}\left|D_{0+}^{\rho} u_{1}(t)\right|+\max _{0 \leqslant t \leqslant 1}\left|u_{1}^{\prime}(t)\right| \leqslant L_{1}, \\
b<\min _{1 / k \leqslant t \leqslant(k-1) / k}\left|u_{2}(t)\right|<\max _{0 \leqslant t \leqslant 1}\left|u_{2}(t)\right| \leqslant r_{2}, \\
\max _{0 \leqslant t \leqslant 1}\left|D_{0+}^{\rho} u_{2}(t)\right|+\max _{0 \leqslant t \leqslant 1}\left|u_{2}^{\prime}(t)\right| \leqslant L_{2}, \quad r_{1}<\max _{0 \leqslant t \leqslant 1}\left|u_{3}(t)\right| \leqslant k b, \\
\min _{1 / k \leqslant t \leqslant(k-1) / k}\left|u_{3}(t)\right|<b, \quad L_{1}<\max _{0 \leqslant t \leqslant 1}\left|D_{0+}^{\rho} u_{3}(t)\right|+\max _{0 \leqslant t \leqslant 1}\left|u_{3}^{\prime}(t)\right| \leqslant L_{2} .
\end{gathered}
$$

The proof is completed. 
Corollary 3.6. If there exist constants $0<r_{1}<b_{1}<k b_{1} \leqslant r_{2}<b_{2}<k b_{2} \leqslant \cdots \leqslant r_{n}$, and $0<L_{1} \leqslant L_{2} \leqslant \cdots \leqslant L_{n-1}, n \in N$, such that $k b_{i} / B \leqslant \min \left\{r_{i+1} / A, L_{i+1} / 2 A, L_{i+1} / 2 M\right\}$, for $1 \leqslant i \leqslant n-1$ and the following conditions are satisfied:

$\left(I_{1}\right) f(t, u, v)<\min \left\{\phi_{p}\left(r_{i} / A\right), \phi_{p}\left(L_{i} / 2 A\right), \phi_{p}\left(L_{i} / M\right)\right\}$ for $(t, u, v) \in[0,1] \times\left[0, r_{i}\right] \times$ $\left[-L_{i}, L_{i}\right]$

(I $) f(t, u, v)>\phi_{p}\left(k b_{i} / B\right)$, for $(t, u, v) \in[1 / k, 1] \times\left[b_{i}, k b_{i}\right] \times\left[-L_{i+1}, L_{i+1}\right]$; then the problem (1.5) has at least $2 n-1$ concave positive solutions.

Proof. If $n=1$, by Condition $\left(I_{1}\right)$ and Step 1 of the proof of Theorem 3.5, we can obtain that $T: \bar{P}\left(\varphi, r_{1} ; \theta, L_{1}\right) \rightarrow P\left(\varphi, r_{1} ; \theta, L_{1}\right) \subset \bar{P}\left(\varphi, r_{1} ; \theta, L_{1}\right)$. From the Schauder fixed-point theorem, the problem (1.5) has at least one fixed-point $u_{1} \in P\left(\varphi, r_{1} ; \theta, L_{1}\right)$.

If $n=2$, by Theorem 3.5, there exist at least three concave positive solutions $u_{2}, u_{3}$, and $u_{4}$. By the induction method, we finish the proof.

Finally, we present an example to check our results.

Example 3.7. Consider the boundary value problem:

$$
\begin{gathered}
D_{0+}^{1 / 2}\left(\phi_{3 / 2}\left(D_{0+}^{5 / 2} u(t)\right)\right)+f\left(t, u(t), D_{0+}^{1 / 2} u(t)\right)=0, \quad 0<t<1, \\
u(0)=u^{\prime}(1)=u^{\prime \prime}(0)=0, \quad D_{0+}^{\alpha} u(t)_{t=0}=0,
\end{gathered}
$$

where

$$
f(t, u, v)= \begin{cases}\frac{t}{20}+6 u^{2}+\left(\frac{|v|}{4 \times 10^{4}}\right)^{4}, & \text { for } u \leqslant 4 \\ \frac{t}{20}+96+\left(\frac{|v|}{4 \times 10^{4}}\right), & \text { for } u>4\end{cases}
$$

Let $k=4$; note that $p=3 / 2, q=3, \alpha=5 / 2, \gamma=1 / 2, \rho=1 / 2$; we have $A \approx 0.9585, B \approx 0.1089$, $M \approx 1.0819$.

Choosing $r_{1}=1 / 4, b=1, r_{2}=10^{4}, L_{1}=2 \times 10^{4}, L_{2}=4 \times 10^{4}$. It is easy to see that $k b / B \leqslant \min \left(r_{2} / A, L_{2} / 2 A, L_{2} / 2 M\right)$, and $f(t, u, v)$ satisfying

(1) $f(t, u, v) \leqslant 0.4875<\min \left\{\phi_{p}\left(r_{1} / A\right), \phi_{p}\left(L_{1} / 2 A\right), \phi_{p}\left(L_{1} / 2 M\right)\right\} \approx 0.5107,(t, u, v) \in$ $[0,1] \times[0,1 / 4] \times\left[-2 \times 10^{4}, 2 \times 10^{4}\right]$,

(2) $f(t, u, v) \geqslant 7.0125>\phi_{p}(k b / B) \approx 6.060,(t, u, v) \in[1 / 4,1] \times[1,4] \times\left[-4 \times 10^{4}, 4 \times 10^{4}\right]$,

(3) $f(t, u, v) \leqslant 97.05<\min \left\{\phi_{p}\left(r_{2} / A\right), \phi_{p}\left(L_{2} / 2 A\right), \phi_{p}\left(L_{2} / 2 M\right)\right\} \approx 102.1419,(t, u, v) \in$ $[0,1] \times\left[0,10^{4}\right] \times\left[-4 \times 10^{4}, 4 \times 10^{4}\right]$. 
By Theorem 3.5, problem (3.21) has at least three concave positive solutions $u_{1}, u_{2}$, and $u_{3}$ satisfying

$$
\begin{gathered}
\max _{0 \leqslant t \leqslant 1}\left|u_{1}(t)\right|<\frac{1}{4}, \max _{0 \leqslant t \leqslant 1}\left|D_{0+}^{\rho} u_{1}(t)\right|+\max _{0 \leqslant t \leqslant 1}\left|u_{1}^{\prime}(t)\right|<2 \times 10^{4}, \\
1<\min _{1 / k \leqslant t \leqslant(k-1) / k}\left|u_{2}(t)\right|<\max _{0 \leqslant t \leqslant 1}\left|u_{2}(t)\right| \leqslant 10^{4}, \\
\max _{0 \leqslant t \leqslant 1}\left|D_{0+}^{\rho} u_{2}(t)\right|+\max _{0 \leqslant t \leqslant 1}\left|u_{2}^{\prime}(t)\right|<4 \times 10^{4}, \\
\frac{1}{4}<\max _{0 \leqslant t \leqslant 1}\left|u_{3}(t)\right| \leqslant 4, \quad \min _{1 / k \leqslant t \leqslant(k-1) / k}\left|u_{3}(t)\right|<1, \\
2 \times 10^{4}<\max _{0 \leqslant t \leqslant 1}\left|D_{0+}^{\rho} u_{3}(t)\right|+\max _{0 \leqslant t \leqslant 1}\left|u_{3}^{\prime}(t)\right|<4 \times 10^{4} .
\end{gathered}
$$

\section{Acknowledgments}

This work was jointly supported by the Natural Science Foundation of Hunan Provincial under Grants 09JJ3005 and 2009JT3042, the Construct Program of the Key Discipline in Hunan Province, and Aid Program for Science and Technology Innovative Research Team in Higher Educational Institutions of Hunan Province.

\section{References}

[1] H. Su, Z. Wei, and B. H. Wang, "The existence of positive solutions for a nonlinear four-point singular boundary value problem with a $p$-Laplacian operator," Nonlinear Analysis: Theory, Methods $\mathcal{E}$ Applications, vol. 66, no. 10, pp. 2204-2217, 2007.

[2] L. Yang, X. P. Liu, and M. Jia, "Multiplicity results for second-order $m$-point boundary value problem," Journal of Mathematical Analysis and Applications, vol. 324, no. 1, pp. 532-542, 2006.

[3] X. G. Zhang and L. S. Liu, "Positive solutions of fourth-order four-point boundary value problems with $p$-Laplacian operator," Journal of Mathematical Analysis and Applications, vol. 336, no. 2, pp. 14141423, 2007.

[4] Y. P. Sun, "Optimal existence criteria for symmetric positive solutions to a three-point boundary value problem," Nonlinear Analysis: Theory, Methods E Applications, vol. 66, no. 5, pp. 1051-1063, 2007.

[5] Z. B. Bai and W. G. Ge, "Existence of three positive solutions for some second-order boundary value problems," Computers E Mathematics with Applications, vol. 48, no. 5-6, pp. 699-707, 2004.

[6] D. Delbosco and L. Rodino, "Existence and uniqueness for a nonlinear fractional differential equation," Journal of Mathematical Analysis and Applications, vol. 204, no. 2, pp. 609-625, 1996.

[7] H. Jafari and V. D. Gejji, "Positive solutions of nonlinear fractional boundary value problems using adomian decomposition method," Applied Mathematics and Computation, vol. 180, no. 2, pp. 700-706, 2006.

[8] J. H. Wang, H. J. Xiang, and Z. G. Liu, "Positive solution to nonzero boundary values problem for a coupled system of nonlinear fractional differential equations," International Journal of Differential Equations, vol. 2010, Article ID 186928, 12 pages, 2010.

[9] R. Dehghani and K. Ghanbari, "Triple positive solutions for boundary value problem of a nonlinear fractional differential equation," Bulletin of the Iranian Mathematical Society, vol. 33, no. 2, pp. 1-14, 2007.

[10] S. Q. Zhang, "Existence of solutions for a boundary value problem of fractional order," Acta Mathematica Scientia, vol. 26, no. 2, pp. 220-228, 2006.

[11] S. Q. Zhang, "Positive solutions for boundary value problems of nonlinear fractional differential equations," Electronic Journal of Differential Equations, vol. 36, pp. 1-12, 2006. 
[12] X. W. Su and L. L. Liu, "Existence of solution for boundary value problem of nonlinear fractional differential equation," Applied Mathematics, vol. 22, no. 3, pp. 291-298, 2007.

[13] Z. B. Bai and H. S. Lü, "Positive solutions for boundary value problem of nonlinear fractional differential equation," Journal of Mathematical Analysis and Applications, vol. 311, no. 2, pp. 495-505, 2005.

[14] M. Benchohra, S. Hamani, and S. K. Ntouyas, "Boundary value problems for differential equations with fractional order and nonlocal conditions," Nonlinear Analysis: Theory, Methods E Applications, vol. 71, no. 7-8, pp. 2391-2396, 2009.

[15] J. H. Wang, H. J. Xiang, and Z. G. Liu, "Positive solutions for three-point boundary values problems of nonlinear fractional differential equations with $p$-Laplacian," Far East Journal of Applied Mathematics, vol. 37, pp. 33-47, 2009.

[16] S. G. Samko, A. A. Kilbas, and O. I. Marichev, Fractional Integrals and Derivatives, Theory and Applications, Gordon and Breach Science, Yverdon, Switzerland, 1993.

[17] I. Podlubny, "Fractional Differential Equations," vol. 198 of Mathematics in Science and Engineering, Academic Press, San Diego, Calif, USA, 1999. 


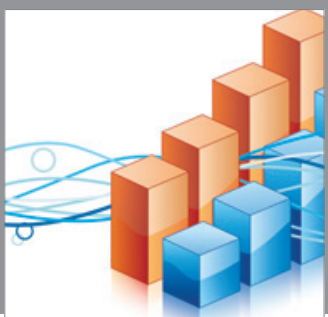

Advances in

Operations Research

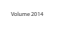

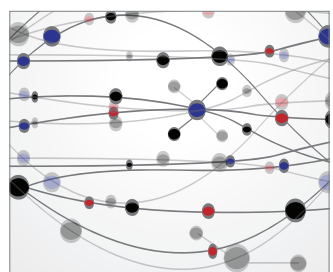

\section{The Scientific} World Journal
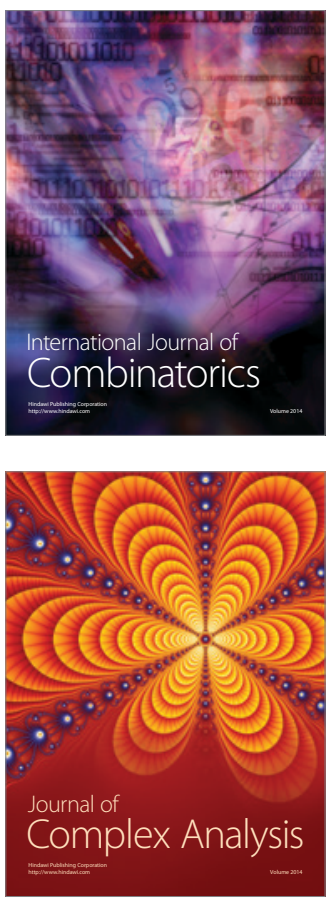

International Journal of

Mathematics and

Mathematical

Sciences
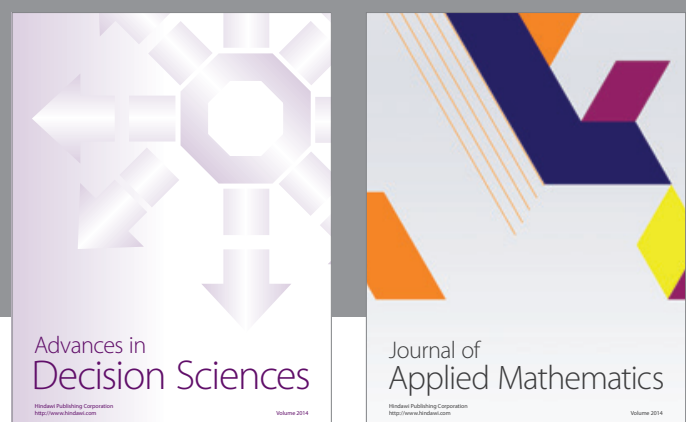

Journal of

Applied Mathematics
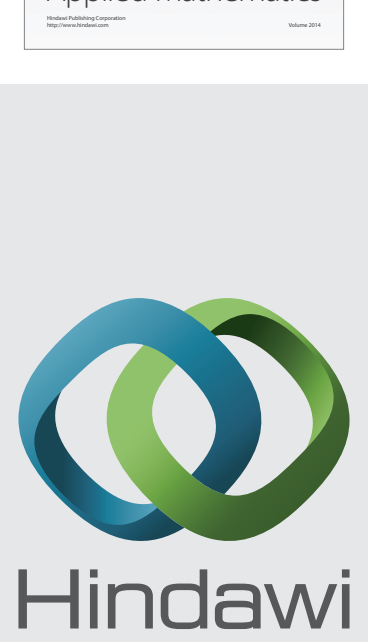

Submit your manuscripts at http://www.hindawi.com
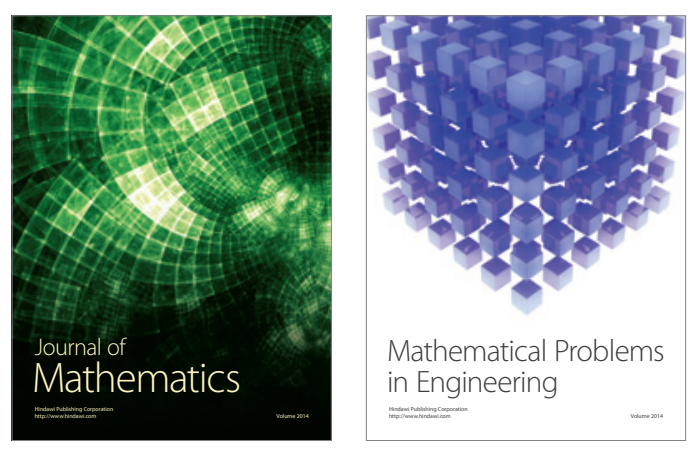

Mathematical Problems in Engineering
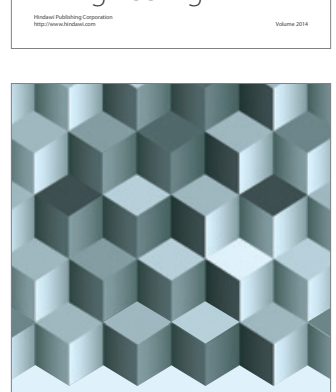

Journal of

Function Spaces
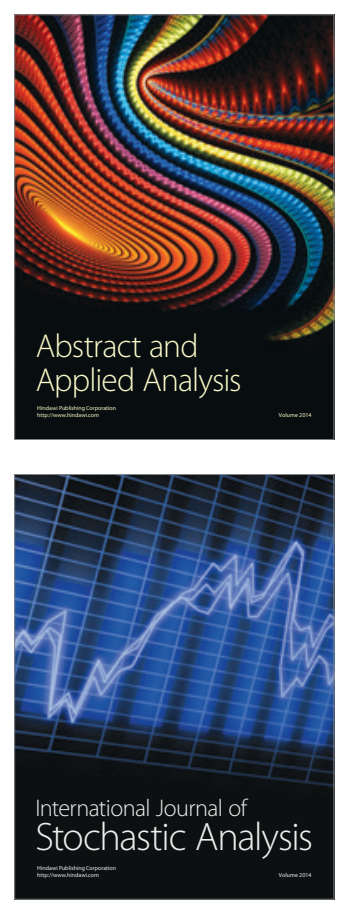

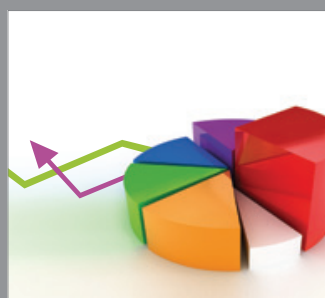

ournal of

Probability and Statistics

Promensencen
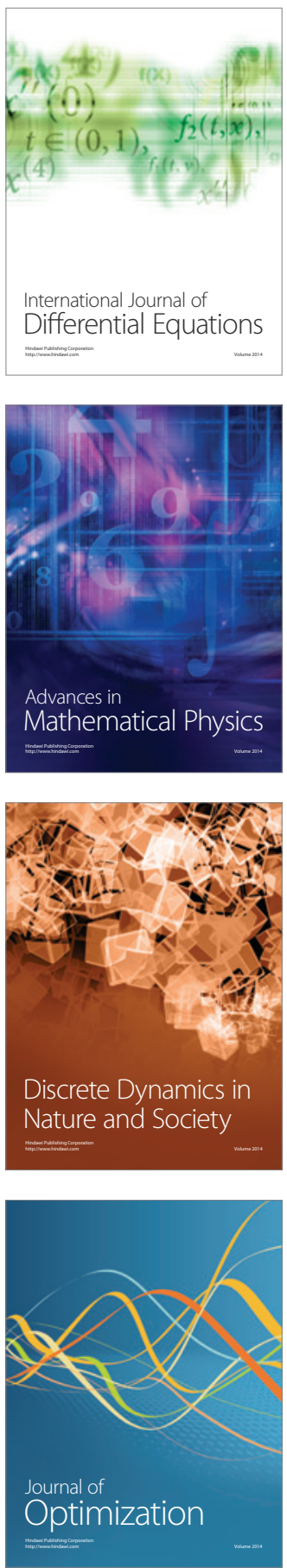